\title{
A Formula to Predict the Magnitude of Achilles Tendon Lengthening Required to Correct Equinus Deformity
}

\author{
Osman Nuri Ozyalvac ${ }^{\mathrm{a}}$ Evren Akpinar ${ }^{\mathrm{a}}$ Volkan Gur $^{\mathrm{b}}$ Kubilay Beng ${ }^{\mathrm{a}}$ \\ Mehmet Firat Yagmurlua Avni Ilhan Bayhan ${ }^{a}$ \\ ${ }^{a}$ Department of Orthopaedic Surgery and Traumatology, Baltalimani Metin Sabanci Bone and Joint Diseases

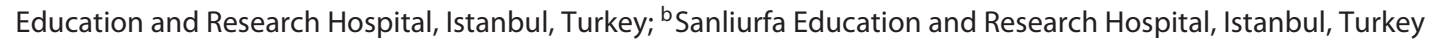

\section{Significance of the Study}

- Achilles tendon lengthening is a frequently used surgical treatment for equinus deformity.

- This study describes a mathematical formula that allows preoperative determination of the amount of Achilles tendon lengthening.

- Calculating the amount of lengthening before surgery will allow more accurate determination of the correct tendon length and will reduce the size of the surgical incision.

\section{Keywords}

Achilloplasty · Achilles tendon lengthening

\begin{abstract}
Objectives: Achilles tendon lengthening (ATL) is one of the most commonly performed procedures in paediatric orthopaedic surgery. An appropriate adjustment of the amount of ATL is crucial to avoid insufficient or excessive lengthening. However, there is currently no effective method to preoperatively calculate the tendon length needed for equinus deformity correction. Thus, in this study we evaluated the accuracy of a calculation using a mathematical model based on the law of cosines. Methods: A total of 16 feet of 14 patients who were scheduled for ATL surgery due to equinus deformity were included in the study. ATL surgery was performed using a standard Z-plasty technique. Calculation of the amount of ATL using the law of cosines, and assessments of intraoperative lengthening of the tendon, were performed
\end{abstract}

\begin{tabular}{ll}
\hline KARGER & $\begin{array}{l}\text { C } 2019 \text { The Author(s) } \\
\text { Published by S. Karger AG, Basel }\end{array}$ \\
E-Mail karger@karger.com & $\begin{array}{l}\text { This is an Open Access article licensed under the Creative Commons } \\
\text { Attribution-NonCommercial-4.0 International License (CC BY-NC) } \\
\text { (http://www.karger.com/Services/OpenAccessLicense), applicable to } \\
\text { the online version of the article only. Usage and distribution for com- } \\
\text { mercial purposes requires written permission. }\end{array}$
\end{tabular}

in a double-blind manner. The extent of lengthening resulting from the two methods was then compared. Results: The mean ATL determined intraoperatively was $23.67 \pm 8.7 \mathrm{~mm}$, and that obtained using the cosine-based method was $22.49 \pm 8.6 \mathrm{~mm}$. Thus, the new method showed excellent statistical agreement with the actual lengthening performed during surgery. Conclusions: The required dimension of ATL can be calculated preoperatively using the mathematical formula presented here. The advantages of this approach are that it allows accurate tendon lengthening and reduces the size of the surgical incision. @ 2019 The Author(s)

Published by S. Karger AG, Basel

\section{Introduction}

Equinus deformity, defined as the inability to dorsiflex the foot beyond plantigrade [1], is a common problem in children with spastic cerebral palsy [2]. Surgical treat- 
ment of equinus deformity consists of Achilles tendon lengthening (ATL) or gastrocnemius recession [3]. The choice between the two methods depends on the results of the Silfverskiöld test [4].

ATL is usually performed on patients with equinus contracture and a negative Silfverskiöld test. Among the techniques often used for ATL are two- or three-cut percutaneous tenotomies and Z-lengthening $[1,5,6]$. However, whereas the lengthening technique has been well studied, the possibility of preoperative determination of the appropriate amount of lengthening has not been considered.

In current practice, the magnitude of ATL is usually established intraoperatively, by positioning the patient's foot at the desired angle of dorsiflexion and then suturing the tendon in the lengthened position. Because determination of the exact tendon length is often difficult, insufficient or excessive ATL may be a major surgical complication. Insufficient lengthening may lead to residual equinus deformity, and excessive lengthening to crouch gait, which occurs due to muscle weakness and is difficult to treat [4]. Furthermore, in the standard Z-plasty technique, the extent of incision cannot be adjusted according to the extent of lengthening, as sufficient tendon contact may not be achieved. A precise ATL procedure is therefore essential [7].

Our hypothesis is that the required magnitude of ATL can be calculated according to the amount of contracture using the law of cosines. The aim of the study was to develop a mathematical equation that determines the appropriate amount of ATL preoperatively.

\section{Patients and Methods}

Equinus deformity was diagnosed in 16 feet of 14 cerebral palsy patients ( 8 boys and 6 girls) and open Z-plasties were planned. Lateral ankle X-ray images with the knee extended and the ankle at maximum dorsiflexion were obtained preoperatively. Patients with a positive Silfverskiöld test established on examination while under anaesthesia, a history of previous and simultaneous surgery for equinus deformity, or selective dorsal rhizotomy were excluded from the study, as were those without appropriate preoperative lateral ankle X-ray images, bone deformity as seen on X-ray, or a neuromuscular disease other than cerebral palsy.

\section{Surgical Technique}

Standard open Z-plasty was performed by the three authors E.A., K.B., and O.N.O. with the patients under general anaesthesia and placed in the supine position. The extent of ATL required was not calculated preoperatively. A tourniquet was used and a posteromedial incision was made. The Achilles tendon was exposed from its insertion in the calcaneus to the proximal muscle belly of

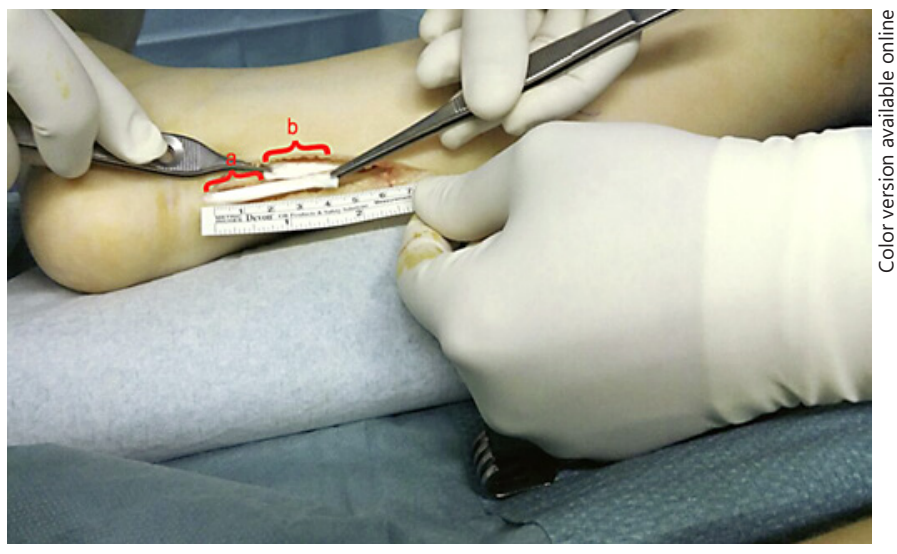

Fig. 1. Measurement during the operation; "a" demonstrates the performed amount of Achilles tendon lengthening, and " $b$ " demonstrates the tendon contact surface area.

the triceps surae, and lengthened while leaving the lateral half of the distal stump and medial half of the proximal junction. ATL and subsequent suturing were performed with the ankle in neutral dorsiflexion.

The tendon limbs were sutured using 3-0 polypropylene $\left(\right.$ Prolene ${ }^{\circledR}$ ) suture material under maximum tension, ensured using Kocher clamps on both limbs according to the Krakow technique. The degree of equinus was measured preoperatively and postoperatively using a goniometer at the angle between the centre line of the calf and the base of the foot. The magnitude of lengthening was determined intraoperatively using a ruler to measure the distance between the tendon stumps (Fig. 1). Changes in the degree of contracture and amount of lengthening were noted. All the ankles were seen to come into neutral dorsiflexion. Postoperative immobilisation was established by 6 weeks in a below-the-knee plaster cast. No complications were encountered during or after surgery.

\section{Calculations}

The rotational centre of the ankle in the sagittal plane (RCA) was designated based on the centre of a circle containing the arc of the talocrural joint surface [8], and the Achilles tendon insertion (AI) as the junction of the posterior border of Kager's triangle with the calcaneus [9]. The distance between the RCA and the AI was defined as "d" (Fig. 2), the amount of change in the angle between the foot and ankle between before and after surgery as " $\alpha$ " (Fig. 3), and the amount of AI displacement after lengthening (which is also the amount of ATL) as " $x$ " (Fig. 3). It was assumed that during ankle plantar dorsiflexion the AI moves in a circle centred at the RCA (Fig. 3).

If $\mathrm{d}$ and $\alpha$ are measured, then the amount of ATL (x) can be calculated using the be simplified as ATL $=\sqrt{ } 2 \mathrm{~d}^{2}(1-\cos (\alpha))$.

In this study, the measurements and calculations were made by a surgeon blinded to the amount of lengthening performed during surgery. The intraoperatively determined ATL is referred to as the performed amount of lengthening (PAL), and the ATL calculated using the above-cited formula as the calculated amount 


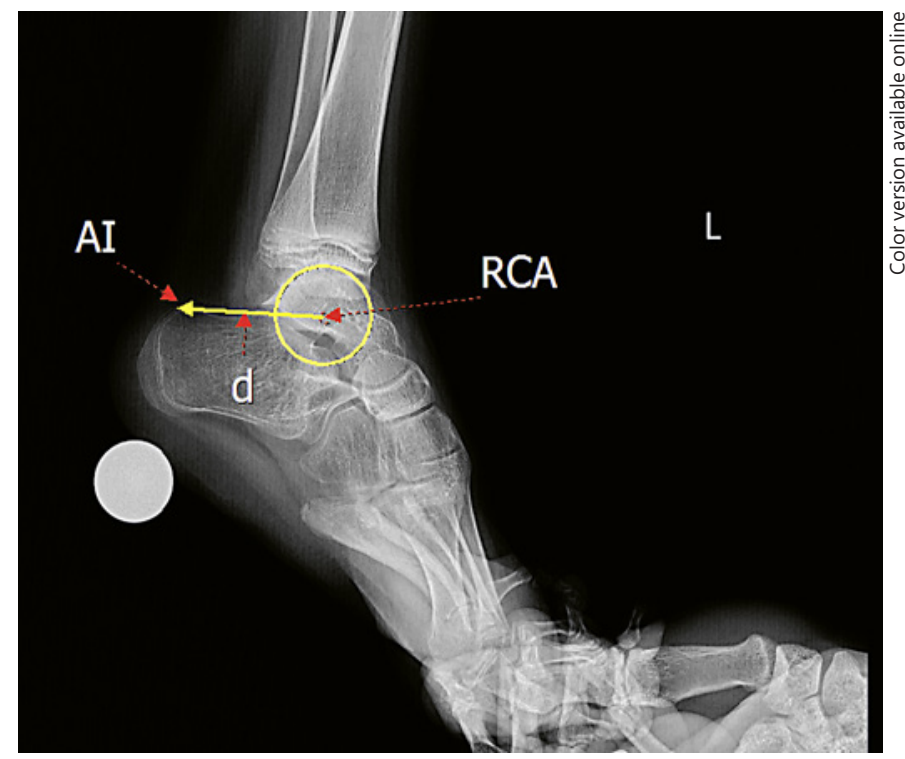

Fig. 2. Identification on $\mathrm{X}$-ray of the rotational centre of the ankle in the sagittal plane (RCA). AI, Achilles tendon insertion; d, distance between the RCA and the AI.

of lengthening (CAL). The agreement between PAL and CAL was assessed.

The distribution of the groups was determined using the Kolmogorov-Smirnov test, and group differences were assessed using the independent-samples $t$ test. Absolute agreement in a two-way mixed random-effects model was used to compute the intraclass correlation coefficient (ICC) based on a $95 \%$ confidence interval (CI). An ICC $\leq 0.5$ indicated a poor fit, an ICC of $0.5-0.75$ a medium fit, an ICC of 0.75-0.90 a good fit, and an ICC $\geq 0.90$ an excellent fit.

\section{Results}

The mean patient age at the time of surgery was $9 \pm$ 3.1 years (range: $6-18$ ). The mean magnitude of lengthening in the PAL and CAL groups was similar $(p=0.728)$ : $23.67 \pm 8.7$ and $22.49 \pm 8.6 \mathrm{~mm}$, respectively. The two groups also showed excellent agreement in the extent of lengthening ( $\mathrm{ICC}=0.953 ; 95 \% \mathrm{CI}$ : 0.861-0.984) (Table 1).

\section{Discussion}

Equinus is a common deformity in children with cerebral palsy [1]. In those with a fixed equinus contracture, it is often treated by ATL. While percutaneous

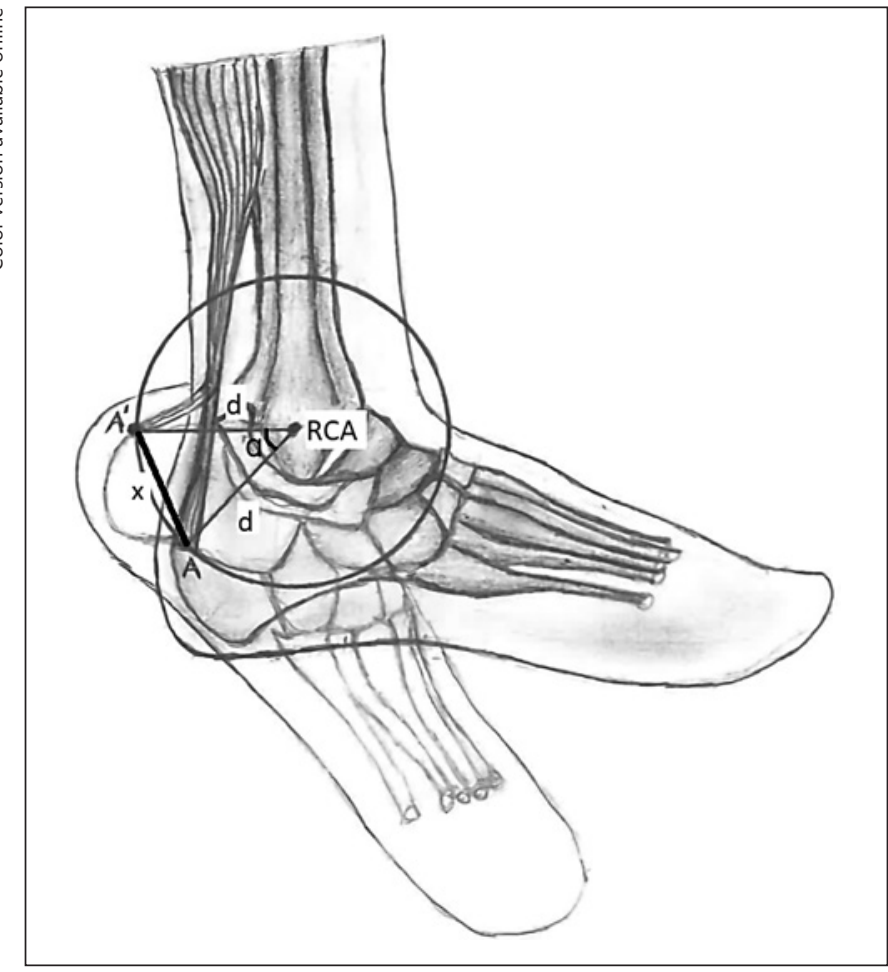

Fig. 3. Displacement of the AI within a circle centred around the RCA during plantar dorsiflexion of the ankle. AI, Achilles tendon insertion; RCA, rotational centre of the ankle in the sagittal plane; $\mathrm{d}$, distance between the RCA and the AI; $\alpha$, amount of change in the equinus between before and after surgery; $\mathrm{x}$, the extent of Achilles tendon lengthening.

procedures are relatively non-invasive, with minimal surgical scarring and morbidity and few wound-healing problems [6], the lack of a method to determine the amount of lengthening required preoperatively increases the risk of over- or underextension of the tendon [5]. Open surgery allows the amount of tendon lengthening to be adjusted during surgery and is therefore not hindered by the inability to determine the required tendon length prior to the procedure. Sufficient durability during tendon repair after lengthening is typically achieved with $3-4 \mathrm{~cm}$ of tendon contact surface area [11].

Since a shorter-than-necessary incision will not allow adequate contact between the tendon limbs, with the standard technique, a long longitudinal incision is made regardless of the extent of the deformity, due to concerns regarding an insufficient tendon contact surface area. The ability to determine the required amount of ATL before surgery will improve the safety of minimally invasive ATL 
Table 1. Correlation between the performed amount of lengthening (PAL) and the calculated amount of lengthening (CAL)

\begin{tabular}{|c|c|c|c|c|c|c|c|}
\hline & \multirow{2}{*}{$\begin{array}{l}\text { Intraclass } \\
\text { correlation }\end{array}$} & \multicolumn{2}{|c|}{$95 \%$ confidence interval } & \multicolumn{4}{|c|}{$F$ test with true value 0} \\
\hline & & lower boundary & upper boundary & value & df1 & df2 & $p$ \\
\hline PAL-CAL & 0.953 & 0.861 & 0.984 & 23.414 & 14 & 14 & $<0.001$ \\
\hline
\end{tabular}

Table 2. Amount of tendon lengthening $(\mathrm{mm})$ required for various degrees of equinus contracture and various distances "d"

\begin{tabular}{lrrrrrrrrr}
\hline & $10^{\circ}$ & \multicolumn{1}{c}{$20^{\circ}$} & \multicolumn{1}{c}{$30^{\circ}$} & \multicolumn{1}{c}{$40^{\circ}$} & \multicolumn{1}{c}{$50^{\circ}$} & $60^{\circ}$ & \multicolumn{1}{c}{$70^{\circ}$} & $80^{\circ}$ & $90^{\circ}$ \\
\hline $10 \mathrm{~mm}$ & 1.74 & 3.47 & 5.18 & 6.84 & 8.45 & 10.00 & 11.47 & 12.86 & 14.14 \\
$20 \mathrm{~mm}$ & 3.49 & 6.95 & 10.35 & 13.68 & 16.90 & 20.00 & 22.94 & 25.71 & 28.28 \\
$30 \mathrm{~mm}$ & 5.23 & 10.42 & 15.53 & 20.52 & 25.36 & 30.00 & 34.41 & 38.57 & 42.43 \\
$40 \mathrm{~mm}$ & 6.97 & 13.89 & 20.71 & 27.36 & 33.81 & 40.00 & 45.89 & 51.42 & 56.57 \\
$50 \mathrm{~mm}$ & 8.72 & 17.36 & 25.88 & 34.20 & 42.26 & 50.00 & 57.36 & 64.28 & 70.71 \\
$60 \mathrm{~mm}$ & 10.46 & 20.84 & 31.06 & 41.04 & 50.71 & 60.00 & 68.83 & 77.13 & 84.85 \\
$70 \mathrm{~mm}$ & 12.20 & 24.31 & 36.23 & 47.88 & 59.17 & 70.00 & 80.30 & 89.99 & 98.99 \\
$80 \mathrm{~mm}$ & 13.94 & 27.78 & 41.41 & 54.72 & 67.62 & 80.00 & 91.77 & 102.85 & 113.14 \\
$90 \mathrm{~mm}$ & 15.69 & 31.26 & 46.59 & 61.56 & 76.07 & 90.00 & 103.24 & 115.70 & 127.28 \\
\hline
\end{tabular}

procedures. For example, a 5 -cm incision will be sufficient for a patient in whom $2 \mathrm{~cm}$ of tendon lengthening and $3 \mathrm{~cm}$ of tendon contact are planned. Alternatively, surgery can be performed in a minimally invasive manner via two incisions spaced $5 \mathrm{~cm}$ apart.

In this study, because our newly devised formula was untested in practice, the amount of lengthening was set using our standard technique and the corresponding calculation was performed after surgery. Analysis of the PAL and CAL data revealed an excellent correlation. Preoperative calculation of the amount of ATL may allow for adequate correction through minimal incision, instead of an incision along the Achilles tendon. The smaller incision will reduce surgical scarring, morbidity, and wound healing problems while shortening the surgical time. Although our calculation cannot be expected to yield an absolute result, as it is a purely geometric approach to a biological problem varying in severity, it does allow preoperative prediction of the magnitude of ATL.

A limitation of this calculation is that it is not a simple mathematical operation, but requires the use of a cosine table. To simplify the calculation, the amount of ATL required for different degrees of equinus contracture and distances " $d$ " is presented in Table 2.

\section{Conclusions}

We developed a formula that determines the amount of ATL needed to correct an equinus contracture. However, further studies are needed to evaluate the clinical outcomes of patients treated with ATL as predicted using this formula.

\section{Statement of Ethics}

This prospective study was approved by the Institutional Review Board of our hospital.

\section{Disclosure Statement}

The authors have no conflicts of interest to disclose. 


\section{References}

1 Shore BJ, White N, Kerr Graham H. Surgical correction of equinus deformity in children with cerebral palsy: a systematic review. J Child Orthop. 2010 Aug;4(4):277-90.

2 Gaines RW, Ford TB. A systematic approach to the amount of Achilles tendon lengthening in cerebral palsy. J Pediatr Orthop. 1984 Aug; 4(4):448-51.

3 Bleck EE. Management of the lower extremities in children who have cerebral palsy. J Bone Joint Surg Am. 1990 Jan;72(1):140-4.
4 Dietz FR, Albright JC, Dolan L. Medium-term follow-up of Achilles tendon lengthening in the treatment of ankle equinus in cerebral palsy. Iowa Orthop J. 2006;26:27-32.

5 Hoffman B, Nunley J. Achilles tendon torsion has no effect on percutaneous triple-cut tenotomy results. Foot Ankle Int. 2006 Nov; 27(11):960-4.

6 Chen L, Ma X, Wang X, Huang J, Zhang C, Wang C. Comparison of four methods for percutaneous Achilles tendon lengthening: a cadaveric study. J Foot Ankle Surg. 2017 MarApr;56(2):271-6.

7 Banks HH, Green WT. The correction of equinus deformity in cerebral palsy. J Bone Joint Surg Am. 1958 Dec;40-A(6):1359-79.
8 Barnett $\mathrm{CH}$, Napier JR. The axis of rotation at the ankle joint in man; its influence upon the form of the talus and the mobility of the fibula. J Anat. 1952 Jan;86(1):1-9.

9 Ly JQ, Bui-Mansfield LT. Anatomy of and abnormalities associated with Kager's fat pad. AJR Am J Roentgenol. 2004 Jan;182(1):14754.

10 Taylor JC. Perioperative planning for twoand three-plane deformities. Foot Ankle Clin. 2008 Mar;13(1):69-121.

11 Karamitopoulos MS, Nirenstein L. Neuromuscular foot: spastic cerebral palsy. Foot Ankle Clin. 2015 Dec;20(4):657-68. 